\title{
AN EXTENSION THEOREM FOR SOBER SPACES AND THE GOLDMAN TOPOLOGY
}

\section{EZZEDDINE BOUACIDA, OTHMAN ECHI, GABRIEL PICAVET, and EZZEDDINE SALHI}

\author{
Received 21 December 2002
}

\begin{abstract}
Goldman points of a topological space are defined in order to extend the notion of prime $G$-ideals of a ring. We associate to any topological space a new topology called Goldman topology. For sober spaces, we prove an extension theorem of continuous maps. As an application, we give a topological characterization of the Jacobson subspace of the spectrum of a commutative ring. Many examples are provided to illustrate the theory.
\end{abstract}

2000 Mathematics Subject Classification: 13C15, 54A10, 54B35, 54F65, 57R30.

1. Introduction. We start by recalling several definitions and notations we will be using in this paper.

If $X$ is a topological space, we denote by $O(X)$ the set of all open subsets of $X$. Recall that a continuous map $g: Y \rightarrow Z$ is said to be a quasihomeomorphism if $U \mapsto g^{-1}(U)$ defines a bijection $\mathcal{O}(Z) \rightarrow \mathscr{O}(Y)$ [9].

A subset $S$ of a topological space $X$ is said to be strongly dense in $X$ if $S$ meets every nonempty locally closed subset of $X$ [9]. Thus a subset $S$ of $X$ is strongly dense if and only if the canonical injection $S \hookrightarrow X$ is a quasihomeomorphism. It is well known that a continuous map $q: X \rightarrow Y$ is a quasihomeomorphism if and only if the topology of $X$ is the inverse image by $q$ of that of $Y$ and the subset $q(X)$ is strongly dense in $Y$ [9].

The notion of quasihomeomorphism is used in algebraic geometry and it has recently been shown that this notion arises naturally in the theory of some foliations associated to closed connected manifolds (see [2, 3]). It is worth noting that quasihomeomorphisms are also linked with sober spaces. Recall that a topological space $X$ is said to be sober if any nonempty irreducible closed subset of $X$ has a unique generic point. Let $X$ be a topological space and $\mathbb{S}(X)$ the set of all irreducible closed subsets of $X$ [9]. Let $U$ be an open subset of $X$ and set $\tilde{U}=\{C \in \mathbb{S}(X) \mid U \cap C \neq \varnothing\}$. Then the collection $(\tilde{U}, U$ is an open subset of $X$ ) provides a topology on $\mathbb{S}(X)$ and the following properties hold [9].

(i) The map

$$
\eta_{X}: X \longrightarrow \mathbb{S}(X), \quad x \longmapsto \overline{\{x\}}
$$

is a quasihomeomorphism. 
(ii) The set $\mathbb{S}(X)$ is a sober space.

(iii) Let $f: X \rightarrow Y$ be a continuous map and let $\mathbb{S}(f): \mathbb{S}(X) \rightarrow \mathbb{S}(Y)$ be the map defined by $\mathbb{S}(f)(C)=\overline{f(C)}$, for each irreducible closed subset $C$ of $X$. Then $\mathbb{S}(f)$ is continuous.

(iv) The topological space $\mathbb{S}(X)$ is called the sobrification of $X$, and the assignment $\mathbb{S}$ defines a functor from the category Top of topological spaces to itself.

(v) If $q: X \rightarrow Y$ is a continuous map, then the following diagram is commutative:

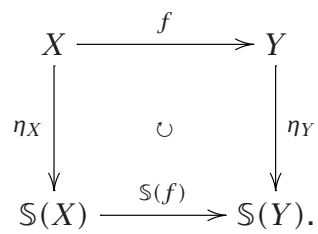

The sobrification serves, sometimes, to give topological characterization of particular spaces (see, e.g., [6]).

Now, we make some observations.

ObSERVATION 1 (General extension theorem of H. Tietze). Let $X$ and $Y$ be two topological spaces. A "general extension theorem for maps of closed subsets of $X$ into $Y$ " is a statement giving conditions on $X$ and $Y$, under which it is true that for every closed $A \subset X$, each continuous $f: A \rightarrow Y$ is extendable over $X$ relative to $Y$. General extension theorems are rare and usually have interesting topological consequences. One can mention then a well-known result of $\mathrm{H}$. Tietze.

THEOREM 1.1 (H. Tietze). Let $X$ be Hausdorff. The following two properties are equivalent:

(1) $X$ is normal;

(2) for every closed $A \subset X$, each continuous $f: A \rightarrow[0,1]$ has a continuous extension $F: X \rightarrow[0,1]$. Furthermore, if $|f(a)|<c$ on $A$, then $F$ can be chosen so that $|F(x)|<c$ on $X$.

The Tietze extension theorem for normal spaces has been improved, by Dugundji [5], using the richer structure of metric spaces.

THEOREM 1.2 (Dugundji [5]). Let $X$ be an arbitrary metric space, $A \subset X a$ closed subset, and $L$ an affine space of type $m$. Then each continuous $f: A \rightarrow L$ has a continuous extension $F: X \rightarrow L$, and in fact $F(X) \subset[$ convex hull of $f(A)]$.

OBSERVATION 2. Let $X$ be a topological space and $\beta X$ the Stone-Čech compactification of $X$. Then every continuous function from $X$ to a compact space $K$ can be extended to $\beta X$. 
The above two observations have a common point: they provide theorems of extension. Our goal is to give such extension theorems for sober spaces. Thus we state the following natural questions.

Problem 1.3. Let $X$ and $Y$ be topological spaces, characterize the continuous maps $q: X \rightarrow Y$, such that for each sober space $Z$ and for each continuous map $f: X \rightarrow Z$, there exists one and only one continuous map $\tilde{f}: Y \rightarrow Z$ making the following diagram commutative:

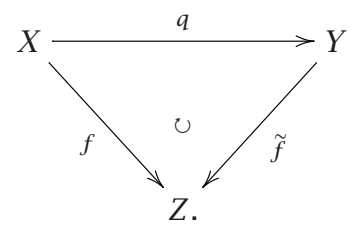

Problem 1.4. Characterize the collection of topological spaces $Z$ such that for each continuous map $q: X \rightarrow Y$ satisfying Problem 1.3 and for each continuous map $f: X \rightarrow Z$, there exists one and only one continuous map $\tilde{f}: Y \rightarrow Z$ such that diagram (1.3) commutes.

The second section deals entirely with the solution of Problems 1.3 and 1.4: quasihomeomorphisms answer Problem 1.3 and sober spaces answer Problem 1.4. Naturally, these answers must provide some interesting applications; these are what we promise in Sections 5 and 6.

A topology $\mathscr{T}$ on a set $X$ is defined to be spectral (and $(X, \mathscr{T})$ is called a spectral space) if the following conditions hold:

(i) $\mathscr{T}$ is sober;

(ii) the quasicompact open subsets of $X$ form a basis of $\mathscr{T}$;

(iii) the family of quasicompact open subsets of $X$ is closed under finite intersections.

In a remarkable paper [10], Hochster has proved that a topological space is homeomorphic to the prime spectrum of some ring if and only if it is a spectral space. In the same paper, Hochster characterizes the maximal prime spectrum of a commutative ring as a quasicompact $T_{1}$-space. Two years later, Hochster gave a topological characterization of the minimal prime spectrum of a ring [11].

Goldman ideals are important objects of investigation in algebra mostly because of their role in the study of graded rings and some applications to algebraic geometry. Thus it is important to pay attention to the Goldman prime spectrum of a ring. Recall that a prime ideal of a commutative ring $R$ is said to be a Goldman ideal (G-ideal) if there exists a maximal ideal $M$ of $R[X]$ such that $p=M \cap R$. If $R$ is an integral domain and (0) is a $G$-ideal, $R$ is called a G-domain.

Over the years, mathematicians have focused attention on $G$-domains; for instance, Goldman [8] and Krull [13] used $G$-ideals for a short inductive proof 
of the Nullstellensatz. The set of all $G$-ideals of a commutative ring $R$ is denoted by $\operatorname{Gold}(R)$ and called the Goldman prime spectrum of that ring.

Recall that a topological space $X$ is said to be a $T_{D}$-space if for each $x \in X$, $\{x\}$ is locally closed.

In [4], Conte has proved that $\operatorname{Spec}(R)$ is a $T_{D}$-space if and only if every prime ideal of $R$ is a $G$-ideal. He has also proved that if $\operatorname{Spec}(R)$ is Noetherian, then $\operatorname{Spec}(R)$ is a $T_{D}$-space if and only if it is finite.

Fontana and Maroscia [7] have also established, by topological methods, several properties of the set of $G$-ideals of a commutative ring. They also discussed in detail a topological approach to a classification of the class of the commutative rings in which every prime ideal is a $G$-ideal.

Note also that rings in which every prime ideal is a $G$-ideal have been studied by Picavet in [15].

By a goldspectral space we mean a topological space which is homeomorphic to some Gold $(R)$. Using the notion of sobrification, Echi has given an intrinsic topological characterization of the Goldman prime spectrum of a commutative ring [6].

Picavet characterized $G$-ideals by a topological property: $p$ is a $G$-ideal of $R$ if and only if $\{p\}$ is locally closed in $\operatorname{Spec}(R)$ (equipped with the Zariski topology) [14]. This characterization motivated us to introduce in Section 3 the notion of a Goldman point in a topological space (a locally closed point).

We also give some characterizations of Goldman points. For instance, $x \in X$ is a $G$-point if and only if $\overline{\{x\}}$ is strongly irreducible. Define $\operatorname{Gold}(X)$ to be the set of all Goldman points of a topological space $X$. When $X$ is $T_{0}$ and has a base of quasicompact open subsets, then $\operatorname{Gold}(X)$ is the smallest strongly dense subset of $X$. Salhi [17] defined a property $(*)$ on topological spaces satisfied by spectra of rings: a topological space $(X, T)$ satisfies $(*)$ if $T$ is compatible with a partial ordering and for every chain $C \subseteq X$ there is some $a \in X$ such that $\overline{\{a\}}=\bar{C}$. If $X$ satisfies $(*)$ and $\operatorname{Gold}(X)=X$, then $X$ is partially well ordered (for the partial ordering induced on $X$ by the topology). Moreover, if the topology on $X$ is compatible with a total ordering and $X$ satisfies $(*)$, then $\operatorname{Gold}(X)=X$ if and only if each nonmaximal element of $X$ has an immediate successor. All these results and others are reminiscents of the theory of $G$-ideals.

Picavet defined a new topology on the spectrum of a ring and called it the Goldman topology or $G$-topology owing to its links with $G$-ideals. This definition can be extended to an arbitrary topological space $(X, T)$, providing a new topology $(X, G)$ called again the Goldman topology or $G$-topology. Moreover, the locally closed subsets in the original topology are a base of open sets for the $G$-topology. If the original topological space is $T_{0}$, the $G$-topology is $T_{2}$; if $X$ is a sober space, the sober subspaces of $(X, T)$ identify with the $G$-closed subsets.

In Section 4, we define and study the $G$-topology on a topological space $X$. When $X$ is the spectrum of a $\operatorname{ring} R$, then $(X, G)$ is homeomorphic to the space 
of minimal prime ideals of a ring $S$. However, we do not know of any natural link between $R$ and $S$. Actually, the $G$-topology on a spectrum has a definition which looks like the patch topology definition of Hochster [10]. More precisely, the $G$-topology is finer than the patch topology. On a spectral space, these topologies are the same if and only if $X$ is a Noetherian space (this is not the case for an arbitrary topological space).

Jacobson topological spaces are also used in algebraic geometry and are linked with quasihomeomorphisms. A topological space $X$ is called a Jacobson space if the set $\mathscr{C}(X)$ of its closed points is strongly dense in $X$. If $X$ is a topological space, we denote by $\operatorname{Jac}(X)$ the set $\{x \in X: \overline{\{x\}}=\overline{\{x\} \cap \mathscr{C}(X)}\}$. It is obviously seen that $\operatorname{Jac}(X)$ is a Jacobson space; we call it the Jacobson subspace of $X$.

Let $R$ be a ring and $\operatorname{Spec}(R)$ its prime spectrum equipped with the Zariski topology. We denote by $\operatorname{Jac}(R)$ the Jacobson subspace of $\operatorname{Spec}(R)$. Following Picavet [14], a prime ideal $p$ of $R$ lies in $\operatorname{Jac}(R)$ if and only if $p$ is the intersection of some maximal ideals of $R$.

By a jacspectral space we mean a topological space homeomorphic to the Jacobson subspace of $\operatorname{Spec}(R)$ for some ring $R$. Section 5 deals with a nice topological characterization of jacspectral spaces: Jacspectral spaces are exactly the quasicompact Jacobson sober spaces.

In Section 6, we collect some examples, showing that many of the results in the earlier sections are best possible.

Note that through this paper, $\subset$ denotes proper containment and $\subseteq$ denotes containment with possible equality. If $\leq$ is an ordering on a set $X$ and $x \in X$, then $[x \uparrow$ [ denotes the set $\{y \in X \mid x \leq y\}$ and $] \downarrow x]$ is the set $\{y \in X \mid y \leq x\}$. Recall that a topology $T$ on a space $X$ is compatible with a partial ordering $\leq$ on $X$ if $\overline{\{x\}}=[x \uparrow[$.

2. An extension theorem for sober spaces. In this section, we look more closely at quasihomeomorphisms.

LEMmA 2.1. Let $q: X \rightarrow Y$ be a quasihomeomorphism. Then the following properties hold:

(1) if $X$ is a $T_{0}$-space, then $q$ is injective;

(2) if $X$ is sober and $Y$ is a $T_{0}$-space, then $q$ is a homeomorphism.

Proof. (1) Let $x_{1}$ and $x_{2}$ be two points of $X$ with $q\left(x_{1}\right)=q\left(x_{2}\right)$. Suppose that $x_{1} \neq x_{2}$, then there exists an open subset $U$ of $X$ such that $x_{1} \in U$ and $x_{2} \notin U$. Since there exists an open subset $V$ of $Y$ satisfying $q^{-1}(V)=U$, we get $q\left(x_{1}\right) \in U$ and $q\left(x_{2}\right) \notin U$, which is impossible. It follows that $q$ is injective.

(2) We start with the obvious observation that if $S$ is a closed subset of $Y$, then $S$ is irreducible if and only if is so $q^{-1}(S)$.

We prove that $q$ is surjective. For this end, let $y \in Y$. According to the above observation, $q^{-1}(\overline{\{y\}})$ is a nonempty irreducible closed subset of $X$. Hence 
$q^{-1}(\overline{\{y\}})$ has a generic point $x$. Thus we have the containments

$$
\overline{\{x\}} \subseteq q^{-1}(\overline{\{q(x)\}}) \subseteq q^{-1}(\overline{\{y\}})=\overline{\{x\}}
$$

Then $q^{-1}(\overline{\{q(x)\}})=q^{-1}(\overline{\{y\}})$. It follows, from the fact that $q$ is a quasihomeomorphism, that $\overline{\{q(x)\}}=\overline{\{y\}}$. Since $Y$ is a $T_{0}$-space, we get $q(x)=y$. This proves that $q$ is a surjective map, and thus $q$ is bijective. One may see that bijective quasihomeomorphisms are homeomorphisms.

THEOREM 2.2. Let $q: X \rightarrow Y$ be a continuous map. Then the following statements are equivalent:

(a) $q$ is a quasihomeomorphism;

(b) $\mathbb{S}(q)$ is a homeomorphism.

PROof. First, we remark that the following diagram is commutative:

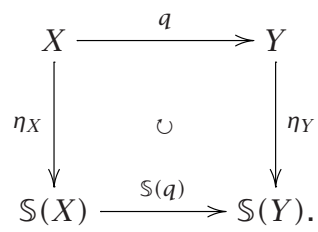

(a) $\Rightarrow$ (b). Since $\eta_{Y} \circ q=\mathbb{S}(q) \circ \eta_{X}$ is a quasihomeomorphism, the map $\mathbb{S}(q)$ is necessarily a quasihomeomorphism. Thus, following Lemma $2.1, \mathbb{S}(q)$ is a homeomorphism.

(b) $\Rightarrow(\mathrm{a})$. Since $\eta_{X}=\left((\mathbb{S}(q))^{-1} \circ \eta_{Y}\right) \circ q$ and $(\mathbb{S}(q))^{-1} \circ \eta_{Y}$ are quasihomeomorphisms, it is easily seen that $q$ is a quasihomeomorphism.

As a consequence of the previous theorem, we state the following one.

THEOREM 2.3 (extension theorem for sober spaces). (1) Let $Z$ be a topological space. Then the following conditions are equivalent:

(i) $Z$ is a sober space;

(ii) for each quasihomeomorphism $q: X \rightarrow Y$ and each continuous map $f: X \rightarrow Z$, there exists one and only one continuous map $F: Y \rightarrow Z$ such that $F \circ q=f$.

(2) Let $q: X \rightarrow Y$ be a continuous map. Then the following conditions are equivalent:

(i) $q$ is a quasihomeomorphism;

(ii) for each sober space $Z$ and each continuous map $f: X \rightarrow Z$, there exists one and only one continuous map $F: Y \rightarrow Z$ such that $F \circ q=f$.

Proof. (1) (i) $\Rightarrow$ (ii). Suppose that such $F$ exists. Then we have $\mathbb{S}(F) \circ \mathbb{S}(q)=$ $\mathbb{S}(f)$. By Theorem 2.2, $\mathbb{S}(q)$ is a homeomorphism, hence $\mathbb{S}(F)=\mathbb{S}(f) \circ(\mathbb{S}(q))^{-1}$. 
On the other hand, the diagram

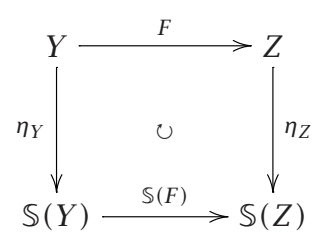

commutes; that is to say, $\eta_{Z} \circ F=\mathbb{S}(F) \circ \eta_{Y}$. Consequently,

$$
F=\left(\eta_{Z}\right)^{-1} \circ \mathbb{S}(F) \circ \eta_{Y}=\left(\eta_{Z}\right)^{-1} \circ \mathbb{S}(f) \circ(\mathbb{S}(q))^{-1} \circ \eta_{Y} .
$$

Thus, it suffices to verify that $F=\left(\eta_{Z}\right)^{-1} \circ \mathbb{S}(f) \circ(\mathbb{S}(q))^{-1} \circ \eta_{Y}$ does the job. Indeed, the following diagram is commutative:

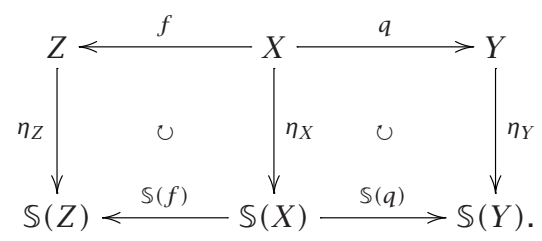

Hence,

$$
\begin{aligned}
F \circ q & =\left(\eta_{Z}\right)^{-1} \circ \mathbb{S}(f) \circ(\mathbb{S}(q))^{-1} \circ \eta_{Y} \circ q \\
& =\left(\eta_{Z}\right)^{-1} \circ \mathbb{S}(f) \circ(\mathbb{S}(q))^{-1} \circ \mathbb{S}(q) \circ \eta_{X} \\
& =\left(\eta_{Z}\right)^{-1} \circ \mathbb{S}(f) \circ \eta_{X} \\
& =\left(\mu_{C}\right)^{-1} \circ \eta_{Z} \circ f=f .
\end{aligned}
$$

(ii) $\Rightarrow$ (i). There exists a unique continuous map $g: \mathbb{S}(Z) \rightarrow Z$ such that the diagram

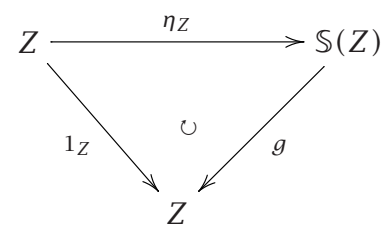

is commutative. Thus the diagram

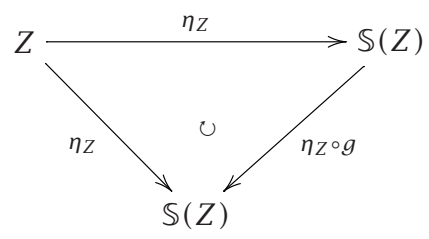


commutes. Hence $\eta_{Z} \circ g=1_{\mathbb{S}(Z)}$ by the implication (i) $\Rightarrow$ (ii). Therefore $\eta_{Z}$ is a homeomorphism and consequently $Z$ is a sober space.

(2) According to part (1), it suffices to show the implication (i) $\Rightarrow$ (ii).

We will prove that $q$ is a quasihomeomorphism. Following Theorem 2.2, it suffices to prove that $\mathbb{S}(q)$ is a homeomorphism.

There exist two morphisms $\widetilde{\eta_{X}}: Y \rightarrow \mathbb{S}(X)$ and $g: \mathbb{S}(Y) \rightarrow \mathbb{S}(X)$ such that the following diagrams commute:
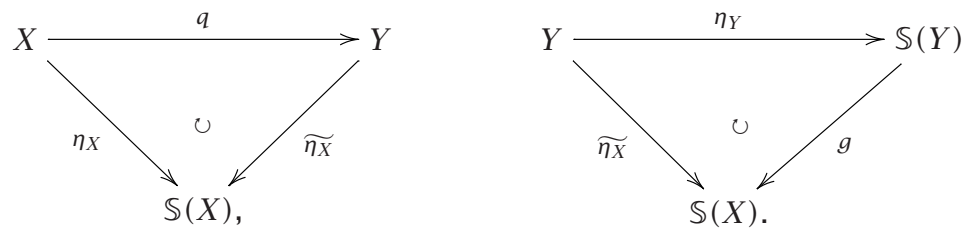

Hence $g \circ \eta_{Y} \circ q=\eta_{X}$. On the other hand, the rectangle (2.2) is commutative. Thus $(g \circ \mathbb{S}(q)) \circ \eta_{X}=g \circ \eta_{Y} \circ q=\eta_{X}$. Hence, using part (1) (i) $\Rightarrow$ (ii), we easily get $g \circ \mathbb{S}(q)=1_{\mathbb{S}(X)}$.

Now, $(\mathbb{S}(q) \circ g) \circ\left(\eta_{Y} \circ q\right)=\mathbb{S}(q) \circ \eta_{X}=\eta_{Y} \circ q$. To prove that $\mathbb{S}(q) \circ g=1_{\mathbb{S}(Y)}$, an analogous reasoning to the previous one is not valid since we have not yet shown that $\eta_{Y} \circ q$ is a quasihomeomorphism. But one can show it by noticing that the following diagrams commute:
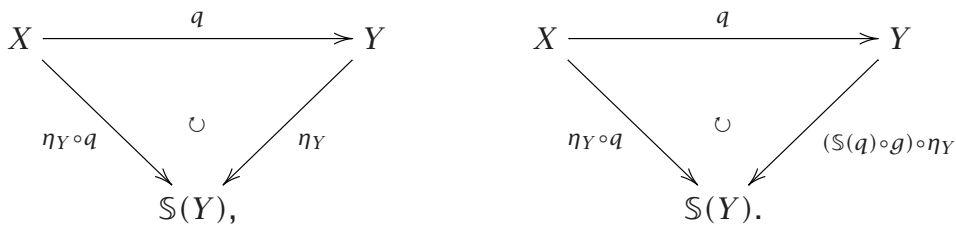

Hence, using part (1) (i) $\Rightarrow($ ii), for the quasihomeomorphism $q$, we get $(\mathbb{S}(q) \circ$ $g) \circ \eta_{Y}=\eta_{Y}$, that is, the diagram

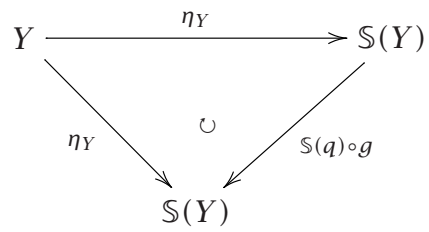

is commutative. Thus, since $\eta_{Y}$ is a quasihomeomorphism, one immediately has $\mathbb{S}(q) \circ g=1_{\mathbb{S}(Y)}$, by part (1) (i) $\Rightarrow$ (ii). This shows that $\mathbb{S}(q)$ is a homeomorphism, so that $q$ is a quasihomeomorphism. 
3. Goldman points. In this section, we define Goldman points in topological spaces in order to recover results about $G$-ideals in a more general setting.

Definition 3.1. Let $X$ be a topological space and $x \in X$. Then $x$ is said to be a $G$-point (Goldman point) of $X$ if $\{x\}$ is a locally closed subset of $X$. We denote by $\operatorname{Gold}(X)$ the set of all $G$-points of $X$.

Proposition 3.2. Let $X$ be a topological space and $x \in X$, then

(i) $x \in \operatorname{Gold}(X)$ if and only if the derived set $\{x\}^{\prime}=\overline{\{x\}} \backslash\{x\}$ is closed;

(ii) $x \notin \operatorname{Gold}(X)$ if and only if $\overline{\{x\}^{\prime}}=\overline{\{x\}}$.

Proof. It is well known that a subset $S$ of a topological space $X$ is locally closed if and only if $\bar{S} \backslash S$ is closed. In particular, $x \in X$ is a $G$-point if and only if $\{x\}^{\prime}$ is closed. Moreover, if $\{x\}^{\prime}$ is not closed, we get $\{x\}^{\prime} \neq \overline{\{x\}}$ so that $\overline{\{x\}^{\prime}}=\overline{\{x\}}$.

Next we give a generalization of a Picavet's result [14, Section I, Proposition 2]. To begin, we need a definition: let $C$ be a closed subset of a topological space $X$; then $C$ is said to be strongly irreducible if for every family $\left\{C_{i}\right\}_{i \in I}$ of closed subsets of $X$, such that $C=\overline{\cup_{i \in I} C_{i}}$, there is some $i \in I$ such that $C=C_{i}$.

Proposition 3.3. Let $X$ be a $T_{0}$-space and $x \in X$. Then $x$ is a $G$-point if and only if $\overline{\{x\}}$ is a strongly irreducible closed subset of $X$.

Proof. Let $X$ be a topological space (not necessarily $T_{0}$ ) and $x \in \operatorname{Gold}(X)$. Suppose that $\overline{\{x\}}=\overline{\cup_{i \in I} C_{i}}$ for some family $\left\{C_{i}\right\}_{i \in I}$ of closed subsets of $X$. Then, assuming that $C_{i} \subset \overline{\{x\}}$ for all $i \in I$, we get $C_{i} \subseteq\{x\}^{\prime}$ so that $\cup_{i \in I} C_{i} \subseteq\{x\}^{\prime}$. Since $\{x\}^{\prime}$ is closed by Proposition 3.2, we get $\overline{\{x\}}=\overline{\cup_{i \in I} C_{i}} \subseteq\{x\}^{\prime}$, contradicting $x \in \overline{\{x\}}$. Therefore, $\overline{\{x\}}$ is a strongly irreducible closed subset of $X$. Conversely, suppose that $\overline{\{x\}}$ is a strongly irreducible closed subset of a $T_{0}$ space $X$. In view of Proposition 3.2, $x \notin \operatorname{Gold}(X)$ gives $\overline{\{x\}^{\prime}}=\overline{\{x\}}$, whence $\overline{\{x\}}=\overline{\cup\left[\{y\} ; y \in\{x\}^{\prime}\right]}$. It follows that $\overline{\{x\}}=\overline{\cup\left[\overline{\{y\}} ; y \in\{x\}^{\prime}\right]}$, from which we deduce that there is some $y_{0} \in\{x\}^{\prime}$ such that $\overline{\left\{y_{0}\right\}}=\overline{\{x\}}$. Since $X$ is a $T_{0}$ space, we get $x=y_{0}$, contradicting $y_{0} \in\{x\}^{\prime}$. Therefore, $x$ is a $G$-point of $X$.

REMARK 3.4. The $T_{0}$ hypothesis cannot be deleted in the above result. Here are two examples.

(1) Take any set $X$ with at least two elements and consider the trivial topology on $X$. Then $\operatorname{Gold}(X)=\varnothing$. Nevertheless, $\overline{\{x\}}$ is a strongly irreducible closed subset of $X$ for each $x \in X$.

(2) Let $X=\{1,2,3,4\}$ and consider the topology defined by $T=\{\varnothing,\{1,2\}$, $X\}$. Then Gold $(X)$ is empty and $\overline{\{x\}}$ is a strongly irreducible closed subset of $X$ for each $x \in X$.

(3) Define $\mathrm{Cl}(x)=\{y \in X \mid \overline{\{x\}}=\overline{\{y\}}\}$ for $x \in X$. In fact, for Proposition 3.3 to hold, we only need to suppose that $\{x\}=\mathrm{Cl}(x)$.

From the definition of strongly dense subsets, it is evident that we have the following proposition. 
Proposition 3.5. Let $X$ be a topological space and $Y$ and $Z$ two subspaces of $X$ such that $X=Y \cup Z$ and $Y \cap Z=\varnothing$.

(1) The set $\operatorname{Gold}(X)$ is contained in $\operatorname{Gold}(Y) \cup \operatorname{Gold}(Z)$.

(2) If, in addition, $Y$ and $Z$ are locally closed, then

(i) $\operatorname{Gold}(X)=\operatorname{Gold}(Y) \cup \operatorname{Gold}(Z)$,

(ii) $\operatorname{Gold}(X)$ is strongly dense in $X$ if and only if $\operatorname{Gold}(Y)$ is strongly dense in $Y$ and $\operatorname{Gold}(Z)$ is strongly dense in $Z$.

Let $R$ be a ring and $\operatorname{Gold}(R)$ the set of all $G$-ideals of $R$. It is well known that $\operatorname{Gold}(R)$ is strongly dense in $\operatorname{Spec}(R)$. Nevertheless, if $X$ is an arbitrary topological space, Gold $(X)$ may be empty (see Remark 3.4 and Example 6.2).

Next we provide examples of topological spaces such that Gold $(X)$ is strongly dense in $X$.

Proposition 3.6. Let $X$ be a $T_{0}$-space.

(1) If $X$ is quasicompact, then there is some closed point $x \in X$.

(2) If $X$ has a base of quasicompact open subsets, then the following properties hold:

(i) $\operatorname{Gold}(X)$ is strongly dense in $X$;

(ii) $\operatorname{Gold}(X)$ is the smallest strongly dense subset of $X$.

Proof. We show (1). Let $\mathscr{C}$ be the family of nonempty closed subsets of $X$. Since $X$ is quasicompact, the ordered set $(\mathscr{b}, \supseteq)$ is inductive, whence $(\mathscr{b}, \subseteq)$ has a minimal element by Zorn's lemma. Let $S$ be a minimal element of $(\mathscr{C}, \subseteq)$, then $\overline{\{x\}}=S$ for each $x \in S$. It follows that $S=\{x\}$ since $X$ is a $T_{0}$-space. Next we show (i). Let $Y=U \cap F$ be a nonempty locally closed subset of $X$, where $U$ is open and $F$ is closed. Let $x \in Y$ and $Q$ a quasicompact open subset such that $x \in Q \subseteq U$. Then $Q \cap F$ is closed in $Q$, whence $Q \cap F$ is quasicompact. According to (1), there exists some $y \in Q \cap F$ such that $\{y\}$ is closed in $Q \cap F$. Thus $\{y\}$ is locally closed in $X$ so that $y \in \operatorname{Gold}(X)$. This proves that $\operatorname{Gold}(X) \cap Y \neq \varnothing$. Hence $\operatorname{Gold}(X)$ is strongly dense in $X$. We prove (ii). Let $Y$ be a strongly dense subset of $X$. If $x \in \operatorname{Gold}(X)$, then $\{x\}$ is a nonempty locally closed subset, whence $\{x\} \cap Y \neq \varnothing$. Therefore, we have $\operatorname{Gold}(X) \subseteq Y$.

COROLlARY 3.7. Let $X$ be a Noetherian $T_{0}$-space. Then Gold $(X)$ is strongly dense in $X$.

We next investigate when $\operatorname{Gold}(X)=X$. We start with a straightforward lemma.

LEMMA 3.8. Let $X$ be a topological space and $Y$ a strongly dense subset of $X$. Then $\operatorname{Gold}(X) \subseteq Y$.

Proposition 3.9. Let $X$ be a topological space. Then the following statements are equivalent:

(1) $\operatorname{Gold}(X)=X$;

(2) the only strongly dense subset of $X$ is $X$. 
Proof. By Proposition 3.6, we get $(1) \Rightarrow(2)$. Conversely, assume that (2) holds and let $x \in X$; then $X \backslash\{x\}$ is not strongly dense in $X$. Hence, there exists some nonempty locally closed subset $Y$ of $X$ such that $Y \cap(X \backslash\{x\})=\varnothing$. Therefore, we get $Y=\{x\}$ so that $x \in \operatorname{Gold}(X)$.

Proposition 3.10. If $X$ is a Noetherian $T_{0}$-space such that $\operatorname{Gold}(X)$ is finite, then $X$ is finite and $\operatorname{Gold}(X)=X$.

Proof. Suppose that $\operatorname{Gold}(X) \neq X$. Define $Y$ to be the set of all closed points of $X$. Then obviously $Y \subseteq \operatorname{Gold}(X)$ so that $Y$ is closed. Since $X \backslash Y$ is a nonempty Noetherian $T_{0}$-space, there exists some $x_{1} \in X \backslash Y$ such that $\left\{x_{1}\right\}$ is a closed point of $X \backslash Y$ (see Proposition 3.6(1)). Hence, $\left\{x_{1}\right\}$ is locally closed in $X$, whence $x_{1} \in \operatorname{Gold}(X)$. Since $\operatorname{Gold}(X) \neq X$, we get that $(X \backslash Y) \backslash\left\{x_{1}\right\}$ is a nonempty open subset of $X \backslash Y$. Arguing as above, we exhibit $x_{2} \in(X \backslash Y) \backslash\left\{x_{1}\right\}$ such that $x_{2} \in \operatorname{Gold}(X)$, and so on. Thus there is an infinite sequence in $\operatorname{Gold}(X)$, contradicting the hypotheses.

REMARK 3.11. Noetherian spectral spaces have properties which do not hold for an arbitrary Noetherian topological space.

(1) In [14, Section II, Corollary of Proposition 3], Picavet proved that when $R$ is a ring with Noetherian spectrum, then $\operatorname{Gold}(R)=\operatorname{Spec}(R)$ if and only if $\operatorname{Spec}(R)$ is finite. This is no longer true for an arbitrary topological space (see Example 6.3).

(2) In the same paper [14], we find that if $R$ is a ring $\operatorname{such}$ that $\operatorname{Spec}(R)=$ $\operatorname{Gold}(R)$, then $(\operatorname{Spec}(R), \subseteq)$ is partially well ordered (i.e., every chain in $\operatorname{Spec}(R)$ is well ordered). This is also no longer true for the partial ordering induced by the topology of an arbitrary $T_{0}$-space (see Example 6.3).

(3) Nevertheless, observe that if $X$ is a $T_{0}$-space such that the closure of each point has finitely many elements, then $\operatorname{Gold}(X)=X$.

Now, we are able to prove that $(X, \leq)$ is partially well ordered if, in addition to $\operatorname{Gold}(X)=X$, we assume that $X$ satisfies property $(*)$ defined below.

Let $(X, T)$ be a topological space. We say that $(X, T)$ satisfies property $(*)$ if $T$ is compatible with a partial ordering $\leq$, and for every chain $C=\left\{a_{i}\right\}_{i \in I} \subseteq X$, there exists some $a \in X$ such that $\overline{\{a\}}=\bar{C}$. Note that the idea of property (*) is due to Salhi (see his work about the space of leaves classes of a foliation [17]). First, we have the following Proposition.

Proposition 3.12. Let $X$ be a $T_{0}$-space and $\leq$ be the partial ordering induced on $X$ by the topology. Let $C=\left\{a_{i}\right\}_{i \in I}$ be a chain in $X$ and $a \in X$. If $\overline{\{a\}}=\bar{C}$, then $a=\inf (C)$.

Proof. From $\overline{\{a\}}=\bar{C}$, we get $a \leq a_{i}$ for every $i \in I$. Now let $b \in X$ such that $b \leq a_{i}$ for each $i \in I$ so that $C \subseteq \overline{\{b\}}$. It follows that $\bar{C}=\overline{\{a\}} \subseteq \overline{\{b\}}$, whence $b \leq a$. Therefore, we have $a=\inf (C)$. 
The following result gives an example of topological spaces satisfying $(*)$.

Proposition 3.13. Let $R$ be a ring. Then the spaces $X=\operatorname{Spec}(R)$ verify $(*)$.

Proof. Let $C=\left\{p_{i}\right\}_{i \in I}$ be a chain of prime ideals of $R$, then $p=\cap\left[p_{i} ; i \in I\right]$ is the infimum of the family $\left\{p_{i}\right\}_{i \in I}$ [12, Theorem 9]. For $x \in A$, consider the special open subset $D(x)=\{q \in \operatorname{Spec}(R) \mid x \notin q\}$ of $\operatorname{Spec}(R)$ containing $p$. Since $x \notin p$, there is some $j \in I$ such that $x \notin p_{j}$ so that $p_{j} \in D(x) \cap C$ and $p$ lies in $\bar{C}$.

REMARK 3.14. There exists a nonspectral topological space satisfying (*) (see Example 6.4).

Proposition 3.15. Let $(X, T)$ be a topological space such that the topology $T$ is compatible with a partial ordering $\leq$. If $X$ satisfies property $(*)$ and $\operatorname{Gold}(X)=X$, then $X$ is partially well ordered.

Proof. Let $C$ be a chain in $(X, \leq)$. Since $X$ satisfies property $(*)$, there is some $x \in X$ such that $x=\inf (C)$ and $\overline{\{x\}}=\bar{C}$ by Proposition 3.12. Then we have $x \in C$, if this does not hold, then for every $y \in C$, we have $x<y$ and $C \subseteq\{x\}^{\prime}$. Now $x \in \operatorname{Gold}(X)$ implies that $\{x\}^{\prime}$ is closed by Proposition 3.2. This leads to a contradiction: $x \in \bar{C} \subseteq\{x\}^{\prime}$. Thus $C$ is well ordered.

Proposition 3.16 generalizes a result of Picavet [14, Section II, Proposition 5] (see also Fontana and Maroscia [7], Ramaswamy and Viswanathan [16]).

A topology $\mathcal{T}$ on $X$ is called a principal topology [18] or a good topology [1] if arbitrary intersections of open subsets of $X$ are open. We review some properties of principal topological spaces studied in [1]. Let $X$ be a set equipped with a binary relation $\mathscr{R}$ and $x \in X$; we denote by $\mathscr{R}_{l}(x)$ the subset of all elements $y \in X$ such that $y=x$ or there exist finitely many elements $x_{1}, x_{2}, \ldots, x_{n}$ such that $x_{1}=y, x_{n}=x$, and $x_{i} \mathscr{\Re} x_{i+1}$ for $i \in\{1, \ldots, n-1\}$. The collection $\left\{\mathscr{R}_{l}(x) \mid x \in X\right\}$ is a base for a topology on $X$ called the left- $\mathscr{R}$-topology and is denoted by $T_{l}(\mathscr{R})$. We have proved that a topological space $(X, T)$ is principal if and only if there exists a binary relation $\mathscr{R}$ on $X$ such that $T$ is the left- $\mathscr{R}$-topology [1]. The following properties hold:

(i) a subset $U$ of $X$ is open in $\left(X, T_{l}(\mathscr{R})\right)$ if and only if $\mathscr{R}_{l}(x) \subseteq U$ for every $x \in U$;

(ii) the closure $\overline{\{x\}}$ of $\{x\}$ is the subset $\mathscr{R}_{r}(x)=\left\{y \in X \mid x \in \mathscr{R}_{l}(y)\right\}$;

Recall that a topology $T$ on $X$ is said to be compatible with the binary relation $\mathscr{R}$ if $\overline{\{x\}}=\mathscr{R}_{r}(x)$ for each $x \in X$.

Proposition 3.16. Let $(X, T)$ be a topological space such that the topology $T$ is compatible with a total ordering $\leq$. If $X$ satisfies Property $(*)$, then the following statements are equivalent:

(1) $\operatorname{Gold}(X)=X$;

(2) every nonmaximal element of $X$ has an immediate successor;

(3) $T$ is a principal topology on $X$. 
Proof. $(1) \Rightarrow(2)$. Let $x$ be a nonmaximal element of $X$, then $\{x\}^{\prime}$ is closed by Proposition 3.2, but $\{x\}^{\prime}$ is a nonempty chain in $X$. We get $\overline{\{x\}^{\prime}}=\overline{\{y\}}$ for some element $y$ by property $(*)$. Thus $y$ is the smallest element of $\{x\}^{\prime}$ since $\{x\}^{\prime}$ is closed. It follows that $y$ is the immediate successor of $x$.

$(2) \Rightarrow(3)$. Let $x$ be an element of $X$. If $x$ is a maximal element of $X$, then ]$\downarrow x]=X$ is an open subset of $X$. When $x$ is not maximal, let $y$ be the immediate successor of $x$. We get $\overline{\{y\}}=[y \uparrow[=X \backslash] \downarrow x]$ since $T$ is compatible with $\leq$. This proves that $] \downarrow x]$ is an open subset of $X$. Therefore, $T$ is a principal topology.

(3) $\Rightarrow(1)$. For all $x \in X$, we have $\{x\}=] \downarrow x] \cap[x \uparrow[=] \downarrow x] \cap \overline{\{x\}}$. It follows that $X=\operatorname{Gold}(X)$.

REMARK 3.17. The total ordering hypothesis is essential in the previous proposition (see Example 6.5).

Proposition 3.18. Let $(X, \leq)$ be a partially ordered set such that each element of $x \in X$ has a finite height ht $(x)$. Assume that $\{x \in X \mid \operatorname{ht}(x)=n\}$ has finitely many elements for each integer $n$. Then $\operatorname{Gold}(X)=X$ for each topology $T$ on $X$ which is compatible with $\geq$.

Proof. Denote by $X_{n}$ the set of all $x \in X$ such that $h t(x) \leq n$. Then $X_{0}$ is finite and $X_{n+1}=X_{n} \cup\left(X_{n+1} \backslash X_{n}\right)$. Moreover, $X_{0}$ is closed since it is a union of finitely many closed points. Suppose that $X_{i}$ is a closed subset when $1 \leq i \leq n$ and set $\left\{x_{1}, \ldots, x_{p}\right\}=X_{n+1} \backslash X_{n}$. Then $\left\{x_{1}, \ldots, x_{p}\right\}$ is a union of closed points in the open set $X \backslash X_{n}$. We get $X_{n+1}=X_{n} \cup\left(X_{n+1} \backslash X_{n}\right)=X_{n} \cup\left\{x_{1}, \ldots, x_{p}\right\}=$ $X_{n} \cup \overline{\left\{x_{1}, \ldots, x_{p}\right\}}$.

It follows that $X_{n+1}$ is a closed subset of $X$. By induction, $X_{n}$ is closed for each integer $n$. Let $x \in X$ such that ht $(x)=n \geq 1$. Then $\{x\}$ is closed in $X \backslash X_{n-1}$. In that case, $x$ belongs to $\operatorname{Gold}(X)$. It follows that $\operatorname{Gold}(X)=X$.

4. Goldman topology. Picavet [14] introduced the Goldman topology (Gtopology) on the spectrum of a ring $R$. This topology is defined as follows. Let $Y$ be a subset of $\operatorname{Spec}(R)$. The closure of $Y$ with respect to the $G$-topology is the set of all prime ideals of $R$ which are an intersection of some elements of $Y$. Then a prime ideal $P$ of $R$ is a $G$-ideal if and only if $P$ is $G$-open.

The family $\{V(I) \cap D(x) \mid I$ is an ideal of $R, x \in R\}$ is a base for the $G^{-}$ topology on $\operatorname{Spec}(R)$. We show that this topology can be defined on any topological space.

Proposition 4.1. Let $X$ be a topological space, $\mathscr{P}(X)$ the set of all its subsets, and $A \in \mathscr{P}(X)$. Set $\bar{A}^{G}=\{x \in X \mid \overline{\{x\}}=\overline{A \cap \overline{\{x\}}}\}$. The map $\mu: \mathscr{P}(X) \rightarrow \mathscr{P}(X)$ defined by $\mu(A)=\bar{A}^{G}$ is a Kuratowski operator closure (thus providing a topology on $X$, called the Goldman topology or G-topology, for short).

Proof. We show four properties:

(1) $\mu(\varnothing)=\varnothing$,

(2) $A \subseteq \mu(A)$ for every $A \in \mathscr{P}(X)$, 
(3) $\mu(\mu(A))=\mu(A)$ for every $A \in \mathscr{P}(X)$,

(4) $\mu(A \cup B)=\mu(A) \cup \mu(B)$ for every $A, B \in \mathscr{P}(X)$.

The first two properties are obvious. Next we prove (3). Using (2), we get $\mu(A) \subseteq \mu(\mu(A))$. Conversely, let $x \in \mu(\mu(A))$ so that $\overline{\{x\}}=\overline{\mu(A) \cap \overline{\{x\}}}$. Let

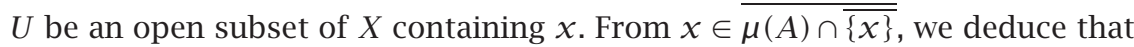

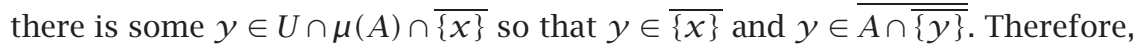
$U \cap(A \cap \overline{\{y\}})$ is nonempty and so is $U \cap(A \cap \overline{\{x\}})$ since $\overline{\{y\}} \subseteq \overline{\{x\}}$. It follows that $x \in \overline{A \cap \overline{\{x\}}}$. Thus we get $\overline{\{x\}}=\overline{A \cap \overline{\{x\}}}$. This proves that $\mu(\mu(A))=$ $\mu(A)$. To end, we show (4). It is enough to see that $\mu(A \cup B) \subseteq \mu(A) \cup \mu(B)$.

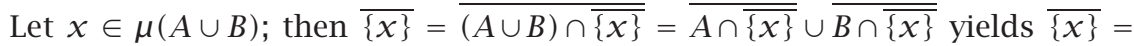
$\overline{A \cap \overline{\{x\}}}$ or $\overline{\{x\}}=\overline{B \cap \overline{\{x\}}}$. Therefore, $x$ lies in $\mu(A) \cup \mu(B)$, which ends the proof.

It is easy to see that the $G$-topology on $\operatorname{Spec}(R)$ is the same as the $G$-topology defined by Picavet [14]. A closed (resp., open) set for the $G$-topology is termed $G$-closed (resp., $G$-open). Therefore, the collection of all $G$-open subsets is $\{X \backslash \mu(A) \mid A \in \mathscr{P}(X)\}$.

Recall that the generization of $Y \subseteq X$ is the subset $g(Y)$ of all $x \in X$ such that $Y \cap \overline{\{x\}} \neq \varnothing$. Moreover, we have $g(Y)=\cap[O ; Y \subseteq O, O$ open $]$.

Proposition 4.2. Let $X$ be a topological space.

(1) If $A \in \mathscr{P}(X)$ is open or closed, then $A$ is $G$-closed.

(2) The family of all locally closed subsets of $X$ is a base for the $G$-topology on $X$. More precisely, $\{U \cap \overline{\{x\}} \mid U$ is open and $x \in U\}$ is a base for the $G$-topology.

(3) The set $g(A)$ is equal to $g\left(\bar{A}^{G}\right)$ and $\bar{A}^{G} \subseteq g(A) \cap \bar{A}$ for all $A \subseteq X$.

Proof. We first prove that every locally closed subset $A$ of $X$ is $G$-open. We only need to show that if $A$ is open or closed, then $A$ is $G$-closed. Let $x \in \bar{A}^{G}$ so that $\overline{\{x\}}=\overline{A \cap \overline{\{x\}}}$. If $A$ is closed, we have $\overline{\{x\}}=A \cap \overline{\{x\}}$, whence $x \in \overline{\{x\}} \subset A$; if $A$ is open, then $A$ meets $\{x\}$ and $x \in A$. In any cases, $x \in A$ and $A=\bar{A}^{G}$. Let $O$ be a $G$-open subset of $X$; we must prove that $O$ is the union of some locally closed subsets of $X$. We set $F=X \backslash O$; let $x$ be an element of $O$ so that $x \notin F=\bar{F}^{G}$; since $x \notin \overline{F \cap \overline{\{x\}}}$, there is some open subset $U_{x}$ containing $x$ and such that $U_{x} \cap F \cap \overline{\{x\}}=\varnothing$, whence $U_{x} \cap \overline{\{x\}} \subseteq O$. Therefore, $O=\cup\left[U_{x} \cap \overline{\{x\}}\right.$; $x \in O$ ]. Now, (3) follows from (1) since $A \subset O \Leftrightarrow \bar{A}^{G} \subset O$ for all open subset $O$.

COROLlary 4.3. Let $X$ be a topological space. A subset $Y$ of $X$ is strongly dense in $X$ if and only if $Y$ is $G$-dense in $X$.

COROLLARY 4.4. Let $R$ be a ring. Then the $G$-closure of $\operatorname{Gold}(R)$ is $\operatorname{Spec}(R)$ and every strongly dense subset of $\operatorname{Spec}(R)$ contains $\operatorname{Gold}(R)$.

Proof. The set $\operatorname{Gold}(R)$ is $G$-dense in $\operatorname{Spec}(R)$ by Corollary 4.3 since $\operatorname{Gold}(R)$ is strongly dense in $\operatorname{Spec}(R)$. A $G$-dense subset $Y$ of $\operatorname{Spec}(R)$ is strongly dense. Hence $Y$ contains Gold $(R)$ by Proposition 3.6. 
REMARK 4.5. The $G$-topology on a topological space $X$ is finer than the initial topology. In particular, for $A \subseteq X$, we have the containments

$$
\stackrel{\circ}{A} \subseteq \stackrel{\circ}{A} \subseteq A \subseteq \bar{A}^{G} \subseteq \bar{A},
$$

where $\stackrel{\circ}{A}, \stackrel{\circ}{A}, \bar{A}^{G}$, and $\bar{A}$ are, respectively, the interior in the initial topology, the interior in the $G$-topology, the closure in the $G$-topology, and the closure in the initial topology.

Proposition 4.6. Let $X$ be a topological space and $a \in X$. Then $\overline{\{a\}}^{G}=$ $\mathrm{Cl}(a)=\{b \in X \mid \overline{\{a\}}=\overline{\{b\}}\}$.

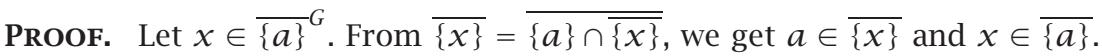
Thus we have $\overline{\{x\}}=\overline{\{a\}}$, that is to say, $x \in \mathrm{Cl}(a)$. The converse is straightforward.

Proposition 4.7. Let $(X, T)$ be a topological space. Denote by $(X, G)$ the space $X$ equipped with the Goldman topology induced by $T$. The following statements are equivalent:

(1) $(X, T)$ is a $T_{0}$-space;

(2) $(X, G)$ is a $T_{2}$-space;

(3) $(X, G)$ is a $T_{1}$-space;

(4) $(X, G)$ is a $T_{0}$-space.

Proof. We first show (1) $\Rightarrow(2)$. Let $x \neq y$ be two elements of $X$. Since $X$ is a $T_{0}$-space, there exists an open subset $U$ of $X$ such that $(x \in U$ and $y \notin U)$ or $(y \in U$ and $x \notin U)$. Set $F=X \backslash U$. We have $U \cap F=\varnothing$ with $(x \in U$ and $y \in F)$ or $(x \in F$ and $y \in U)$. Therefore, the $G$-topology is Hausdorff. Now we show (4) $\Rightarrow(1)$. Let $a$ and $b$ be two elements of $X$ such that $\overline{\{a\}}=\overline{\{b\}}$ so that $\mathrm{Cl}(a)=\mathrm{Cl}(b)$. In view of Proposition 4.6, we get $\overline{\{a\}}^{G}=\overline{\{b\}}^{G}$. Since $(X, G)$ is a $T_{0}$-space, we have $a=b$. Therefore, $(X, T)$ is a $T_{0}$-space.

REMARK 4.8. It may be checked easily that the following properties hold.

(1) If $(X, T)$ is a $T_{1}$-space, then $(X, G)$ is a discrete topological space.

(2) The set $(X, G)$ is a discrete topological space if and only if $X=\operatorname{Gold}(X)$. In particular, if $(X, G)$ is Hausdorff and nondiscrete, $(X, T)$ is a $T_{0}$-space and not a $T_{1}$-space.

(3) Every locally closed subset $A$ of $X$ is a clopen set in $(X, G)$ and satisfies $A=\cup[\mathrm{Cl}(a) ; a \in A]$.

Proposition 4.9. Let $(X, T)$ be a topological space. If the $G$-topology on $X$ is quasicompact, then the topological space $(X, T)$ is Noetherian.

Proof. Let $U$ be an open subset of $X$. Because $U$ is $G$-closed by Proposition 4.2, $U$ is $G$-quasicompact. Then $U$ is quasicompact since the $G$-topology on $X$ is finer than the original topology on $X$. Therefore, $X$ is a Noetherian space. 
REMARK 4.10. The converse of the above proposition is not true (see Example 6.3). Nevertheless, things are nicer for a spectral space.

The family of all closed subsets and all quasicompact open subsets of $X=$ $\operatorname{Spec}(R)$ is a subbase of closed sets for a topology called the patch topology on $X$ [10]. The family of all quasicompact open subsets and their complements is an open subbase for the patch topology. A patch in $X$ is a closed set for the patch topology. It is easily seen that the $G$-topology is finer than the patch topology.

Proposition 4.11. Let $X$ be a spectral space. Then the following statements are equivalent:

(1) the $G$-topology on $X$ is compact;

(2) $X$ is a Noetherian space;

(3) the G-topology on $X$ coincides with the patch topology on $X$.

Proof. Thanks to [14, Section I, Proposition 4], (2) and (3) are equivalent. Then $(1) \Rightarrow(2)$ follows from Proposition 4.9 and $(3) \Rightarrow(1)$ is a consequence of the compactness of the patch topology on $X[10]$.

Proposition 4.12. Let $(X, T)$ be a topological space and $Y \subseteq X$. Then $Y$ is strongly dense in $\bar{Y}^{G}$ (for the topologies induced by $T$ ).

Proof. Let $Z^{\prime}=Z \cap \bar{Y}^{G}$ be a nonempty locally closed subset of $\bar{Y}^{G}$, where $Z$ is a locally closed subset of $X$. Since $Z$ is $G$-open, we get $Z^{\prime} \cap Y \neq \varnothing$ by ${\overline{Z^{\prime}}}^{G}=\overline{Z \cap Y}^{G}$. Hence $Y$ is strongly dense in $\bar{Y}^{G}$.

Proposition 4.13. Every $G$-closed subset of a spectral space $X$ has at least a minimal element for the natural order defined by the topology (more precisely, every $G$-closed subset of $X$ is $\geq$-inductive).

Proof. Let $X=\operatorname{Spec}(R)$, where $R$ is a ring, and $Y \subseteq X$ a $G$-closed subset. Let $\left\{p_{i} \mid i \in I\right\} \subseteq Y$ be a nonempty chain and $p$ the intersection of all $p_{i}$. Since $\bar{Y}^{G}$ is the set of all prime ideals of $R$ which are an intersection of some prime ideals belonging to $Y$, we see that $p \in Y$. Therefore $(Y, \supseteq)$ is inductive.

REMARK 4.14. The above proposition does not hold for an arbitrary $T_{0}$ space (see Example 6.3).

Proposition 4.15. Let $R$ be a ring. Every patch of $\operatorname{Spec}(R)$ is an intersection of Zariski quasicompact $G$-open sets of $\operatorname{Spec}(R)$.

Proof. According to [10, Section 7], a patch set is an intersection of constructible subsets. Moreover, a constructible subset is a union of finitely many locally closed quasicompact subsets.

THEOREM 4.16. Let $X$ be a $T_{0}$-space.

(1) If $X$ is a sober space, every $G$-closed subset of $X$ is sober.

(2) Every sober subspace of $X$ is a $G$-closed subset of $X$. 
Proof. We show (1). Let $F$ be an irreducible closed subset of a $G$-closed subset $Y$ of $X$. We have $F=Y \cap \bar{F}$. Since $F$ is irreducible in $Y$, so is $F$ in $X$. Hence $\bar{F}$ is an irreducible closed subset of $X$. Therefore, $\bar{F}$ has a generic point $x$, that is to say, $\bar{F}=\overline{\{x\}}$ because $X$ is sober. It follows that $F=Y \cap \overline{\{x\}}$ and $\bar{F}=\overline{\{x\}}=\overline{Y \cap \overline{\{x\}}}$. This implies that $x \in Y$ by $G$-closeness of $Y$. Moreover, we have $x \in Y \cap \bar{F}=F$, which proves that $x$ is the generic point of $F$ (in the subspace $Y$ ). Now we prove (2). Let $Y$ be a sober subspace of $X$ and $x \in \bar{Y}^{G}$,

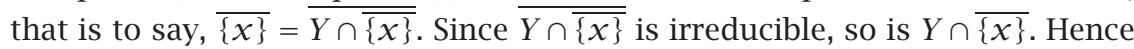
$Y \cap \overline{\{x\}}$ has a generic point $y \in Y \cap \overline{\{x\}}$ so that $Y \cap \overline{\{x\}}=Y \cap \overline{\{y\}}$. Moreover, $x$ and $y$ belong to $\bar{Y}^{G}$. This yields $\overline{\{x\}}=\overline{\{y\}}$. It follows that $x=y \in Y$ since $X$ is a $T_{0}$-space. Therefore, $Y$ is a $G$-closed subset of $X$.

REMARK 4.17. In view of Theorem 4.16, the $G$-closed subsets of $X$ are the sober subspaces of $X$ when $X$ is a sober space. Nevertheless, when $X$ is a $T_{0}$ space, a $G$-closed subset of $X$ need not be sober (see Example 6.3).

Proposition 4.18. Let $X$ be a $T_{0}$-space and $Y$ a $G$-dense subset. Then, for each $x \in X, x=\inf (\overline{\{x\}} \cap Y)$ ( $X$ is equipped with the partial ordering $\leq$ induced by the topology).

Proof. For each $y \in \overline{\{x\}} \cap Y$, we have $y \in \overline{\{x\}}=[x \uparrow[=\{z \in X / x \leq z\}$, whence $x \leq y$. Let $z \in X$, such that $z \leq y$ for any $y \in Y \cap \overline{\{x\}}$, and assume that $x \notin \overline{\{z\}}$. Then $\overline{\{x\}} \cap(X \backslash \overline{\{z\}})$ is nonempty and locally closed. Since $Y$ is strongly dense, we get $\overline{\{x\}} \cap(X \backslash \overline{\{z\}}) \cap Y \neq \varnothing$. Hence, there exists some $y \in \overline{\{x\}} \cap Y$ such that $y \in X \backslash \overline{\{z\}}$, that is to say, $z \nless y$; this is a contradiction. It follows that $z \leq x$, proving that $x=\inf (\overline{\{x\}} \cap Y)$.

We recall some topological definitions and link them with the $G$-topology. Let $\operatorname{Min}(R)$ denote the set of all minimal prime ideals of a ring $R$ equipped with the relativization of the Zariski topology. It is well known that $\operatorname{Min}(R)$ is Hausdorff.

According to Hochster, a topological space $X$ is said to be minspectral if there is some $\operatorname{ring} R$ such that $X$ is homeomorphic to $\operatorname{Min}(R)$ [11]. As in [11], an $m$-base $\mathscr{P}$ for a Hausdorff space $X$ is a base of open sets such that every subset $S$ of $\mathscr{B}$ which has the finite intersection property has nonempty intersection.

A topology is said to be scattered if every point has a base of clopen neighborhoods. Such a topology is totally disconnected. A Hausdorff space $X$ is said to be completely regular (or Tychonoff) if for each point $x \in X$ and every closed subset $V$ of $X$ such that $x \notin V$, there is a continuous map $\varphi: X \rightarrow[0,1]$ such that $\varphi(x)=1$ and $\varphi(a)=0$ for every $a \in V$.

Proposition 4.19. Let $X$ be a topological space.

(1) If $X$ is a spectral space, then the $G$-topology on $X$ is minspectral.

(2) If $X$ is a $T_{0}$-space, then the $G$-topology on $X$ is scattered.

(3) Every scattered topology is completely regular. In particular the G-topology on a $T_{0}$-space is completely regular. 
Proof. With the hypothesis of (1) being granted, the $G$-topology on $X$ is Hausdorff since $X$ is $T_{0}$. Let $\mathscr{B}$ be the family of all subsets $Y$ of $X$ such that $Y=F \cap O$, where $F$ is closed and $O$ is quasicompact open. Then $\mathscr{B}$ is a base for the $G$-topology and its elements are patches. Since the patch topology is compact [10], $\mathscr{B}$ is an $m$-base. Thanks to Hochster's result [11, Theorem 1], the $G$-topology on $X$ is minspectral. We show (2). Every locally closed subset is $G$-open and $G$-closed. Let $x \in X$, then the family of locally closed subsets of $X$ containing $x$ is a base of $G$-clopen neighborhoods of $x$. Now, (3) is a consequence of the following considerations. Let $X$ be a scattered topological space, $x \in X$, and $V$ a closed subset of $X$ such that $x \notin V$. There exists a clopen subset $O$ of $X$ containing $x$ such that $O \subseteq X \backslash V$. Since $\{O, X \backslash O\}$ is an open covering of $X$, we can define a continuous map $\varphi: X \rightarrow[0,1]$ such that $\varphi(O)=\{1\}$ and $\varphi(X \backslash O)=\{0\}$. We have thus checked out the three conditions.

THEOREM 4.20. Let $X$ be a sober topological space and $Y \subseteq X$. Then $\bar{Y}^{G}$ is homeomorphic to $\mathbb{S}(Y)$.

Proof. In view of Theorem $4.16, \bar{Y}^{G}$ is a sober space so that $\bar{Y}^{G} \simeq \mathbb{S}\left(\bar{Y}^{G}\right)$. On the other hand, the inclusion $Y \rightarrow \bar{Y}^{G}$ is a quasihomeomorphism (see Proposition 4.12). Hence $\mathbb{S}(Y) \simeq \mathbb{S}\left(\bar{Y}^{G}\right)$ by Theorem 2.2. Therefore $\bar{Y}^{G}$ is homeomorphic to $\mathbb{S}(Y)$.

Proposition 4.21. Let $X$ be a Noetherian $T_{0}$-topological space.

(1) The canonical injection $X \rightarrow \mathbb{S}(X)$ identifies $X$ with a subspace of $\mathbb{S}(X), X$ is strongly dense in $\mathbb{S}(X)$ and $\mathbb{S}(X)$ is a Noetherian spectral space. In particular, the $G$-topology on $\mathbb{S}(X)$ is the patch topology and induces the $G$-topology on $X$.

(2) The set $\operatorname{Gold}(X)$ is equal to $\operatorname{Gold}(\mathbb{S}(X))$.

Proof. Let $X$ be a Noetherian topological $T_{0}$-space. Then its sobrification $\mathbb{S}(X)$ is a Noetherian spectral space. Indeed, $\mathbb{S}(X)$ is Noetherian whenever $X$ is Noetherian [9, Chapter 0 , Section 3, Proposition 7.6]. In this case, $\mathbb{S}(X)$ is Noetherian and sober, whence a spectral space. Then $X$ can be identified with a subspace of $\mathbb{S}(X)$ and $X$ is strongly dense in $\mathbb{S}(X)$ by [9, Chapter 0 , Section 3, Proposition 7.1(b)]. We get Gold $(\mathbb{S}(X)) \subseteq X$ by Proposition 3.6 because $X$ is strongly dense. It follows that $\operatorname{Gold}(\mathbb{S}(X)) \subseteq \operatorname{Gold}(X)$. Now $\operatorname{Gold}(\mathbb{S}(X))$ is strongly dense in $X$ so that $\operatorname{Gold}(X)=\operatorname{Gold}(\mathbb{S}(X))$.

5. A topological characterization of the Jacobson prime spectrum of a commutative ring. Recall that a topological space $X$ is said to be a Jacobson space if the set $\mathscr{C}(X)$ of all closed points of $X$ is strongly dense in $X$ [9, Chapter 0, Section 3, Proposition 8.1]. Therefore, $X$ is a Jacobson space if and only if $X=\overline{\mathscr{C}(X)}^{G}$. Obviously, when $X$ is a topological space, $\operatorname{Jac}(X)=\overline{\mathscr{C}(X)}^{G}$ is a Jacobson space; we call it the Jacobson subspace of $X$.

Let $R$ be a ring. We denote by $\operatorname{Jac}(R)$ the Jacobson subspace of $\operatorname{Spec}(R)$. Following Picavet [14], a prime ideal $p$ of $R$ is in $\operatorname{Jac}(R)$ if and only if $p$ is the 
intersection of all maximal ideals $m$ of $R$ such that $p \subseteq m$. A jacspectral space is defined to be a topological space homeomorphic to the Jacobson space of $\operatorname{Spec}(R)$ for some ring $R$.

We aim to give a topological characterization of jacspectral spaces by using our previous results. We need a lemma.

LEMMA 5.1. Let $f: Y \rightarrow Z$ be a quasihomeomorphism. If $U$ is an open subset of $Z$, then the following statements are equivalent:

(i) $U$ is quasicompact;

(ii) $f^{-1}(U)$ is quasicompact.

Denote by $O(X)$ the set of all open subsets of a topological space $X$. Then the proof of Lemma 5.1 is an easy consequence of the following fact: $U \mapsto f^{-1}(U)$ defines a bijection $\mathbb{O}(Z) \rightarrow \mathcal{O}(Y)$.

We now head towards an important result which completely characterizes jacspectral spaces.

THEOREM 5.2. Let $X$ be a topological space. The following statements are equivalent:

(1) $X$ is a jacspectral space;

(2) $X$ is a quasicompact Jacobson sober space.

Proof. We first prove $(1) \Rightarrow(2)$. We need only to show that the Jacobson space of a spectral space is a quasicompact Jacobson sober space. Let $R$ be a ring and $X=\operatorname{Jac}(R)$ the Jacobson space of $\operatorname{Spec}(R)$. As we have seen, $X$ is a Jacobson space. Since $X=\overline{\operatorname{Max}(R)}^{G}$ and $\operatorname{Spec}(R)$ is a sober space, $X$ is sober by Theorem 4.16. It is well known that $\operatorname{Max}(R)$ is quasicompact [10]. Moreover, the canonical injection $\operatorname{Max}(R) \hookrightarrow \overline{\operatorname{Max}(R)}^{G}=X$ is a quasihomeomorphism since $\operatorname{Max}(R)$ is strongly dense in $\overline{\operatorname{Max}(R)}^{G}$ (see Proposition 4.12). Hence $X$ is quasicompact by Lemma 5.1 . Next we show that $(2) \Rightarrow(1)$. Suppose that $X$ is a quasicompact Jacobson sober space and let $\mathscr{C}(X)$ be the set of all its closed points. We have $X=\overline{\mathscr{C}(X)}^{G}$. Consequently, the canonical injection $\mathscr{C}(X) \hookrightarrow X$ is a quasihomeomorphism, whence $\mathscr{C}(X)$ is quasicompact by Lemma 5.1 . Observe that $\mathscr{C}(X)$ is a $T_{1}$-space. Therefore, there exists some ring $R$ such that $\mathscr{C}(X)$ is homeomorphic to $\operatorname{Max}(R)$ (see Hochster [10]). Let $\varphi: \mathscr{C}(X) \rightarrow \operatorname{Max}(R)$ be a homeomorphism and $i: \operatorname{Max}(R) \rightarrow \operatorname{Jac}(R)$ the canonical injection. Then $f=i \circ \varphi: \mathscr{C}(X) \rightarrow \operatorname{Jac}(R)$ is a quasihomeomorphism. In view of Theorem 2.3, there exists a continuous extension $\tilde{f}: X \rightarrow \operatorname{Jac}(R)$. This extension is also a quasihomeomorphism. Now, since $X$ is sober and $\operatorname{Jac}(R)$ is a $T_{0}$-space, $\tilde{f}$ is a homeomorphism by Lemma 2.1.

Our next concern will be the construction of jacspectral spaces from Jacobson quasicompact spaces. First, we need some preliminary results.

Proposition 5.3. (1) Let $q: X \rightarrow Y$ be an injective quasihomeomorphism, then $\mathscr{b}(Y) \subseteq q(\mathscr{C}(X))$. 
(2) Let $X$ be a $T_{0}$-space. Then $q(\mathscr{b}(X))=\mathscr{b}(\mathbb{S}(X))$, where $q: X \rightarrow \mathbb{S}(X)$ is the injection of $X$ onto its sobrification $\mathbb{S}(X)$.

(3) Let $q: X \rightarrow Y$ be a quasihomeomorphism and $S$ a subset of $X$. Then the following statements are equivalent:

(i) $S$ is strongly dense in $X$;

(ii) $q(S)$ is strongly dense in $Y$.

Proof. (1) The proof is straightforward.

(2) Following (1), the proof will be complete if we show that $q(\mathscr{C}(X)) \subseteq$ $\mathscr{C}(\mathbb{S}(X))$.

Let $x \in \mathscr{b}(X)$. We claim that $\overline{\{q(x)\}}=\{q(x)\}$.

Let $F \in \overline{\{q(x)\}}$. We must prove that $F=q(x)=\overline{\{x\}}=\{x\}$.

First, we observe that $x \in F$. To see this, suppose that $x \notin F$, then $F \in \tilde{U}$, where $U=X-\{x\}$. Since $F \in \overline{\{q(x)\}}, q(x) \in \widetilde{U}$, hence $q(x) \cap U \neq \varnothing$. This yields $x \in U$, a contradiction. It follows that $x \in F$.

Let $y \in F$. Suppose that $y \neq x$, then $F \cap U \neq \varnothing$, where $U=X-\{x\}$. The rest of the proof runs as before, proving that $y=x$. Therefore $q(x) \in \mathscr{C}(\mathbb{S}(X))$.

(3) The proof is straightforward.

Next, we derive a useful tool for constructing jacspectral spaces.

COROLlaRY 5.4. Let $X$ be a $T_{0}$-space. Then the following statements are equivalent:

(i) $X$ is a quasicompact Jacobson space;

(ii) $\mathbb{S}(X)$ is a jacspectral space.

Proof. We start with the following two observations.

(i) Let $q: X \rightarrow \mathbb{S}(X)$ be the injection of $X$ onto its sobrification $\mathbb{S}(X)$. Following Proposition 5.3, $X$ is a Jacobson space if and only if $\mathbb{S}(X)$ is.

(ii) The equivalence, $X$ is quasicompact if and only if $\mathbb{S}(X)$ is, follows immediately from Lemma 5.1.

Therefore, if $X$ is a quasicompact Jacobson space, then $\mathbb{S}(X)$ is a sober quasicompact Jacobson space, and according to Theorem $5.2, \mathbb{S}(X)$ is a jacspectral space. Conversely, if $\mathbb{S}(X)$ is jacspectral, then it is a quasicompact Jacobson space, and the above observations imply that $X$ is so.

COROLlary 5.5. Let $X$ be a Noetherian $T_{0}$-space. Then $\mathbb{S}(\operatorname{Jac}(X))$ is a jacspectral space.

COROLlaRY 5.6. Let $X$ and $Y$ be two $T_{0}$-spaces and $q: X \rightarrow Y$ a quasihomeomorphism. Then $X$ is a quasicompact Jacobson space if and only if is so $Y$.

COROLLARY 5.7. Let $X$ be a $T_{0}$-space. Then the following statements are equivalent:

(i) $X$ is a quasicompact Jacobson space;

(ii) $X$ is injected by a quasihomeomorphism into a jacspectral space. 
6. Examples. Recall that a topological space is said to be principal if every intersection of open subsets of $X$ is open $[1,18]$. Principal topological spaces provide examples of $G$-points and $G$-topologies. The left topology associated to a partially ordered set is principal and every element of $X$ is a $G$-point. Nevertheless, there exists a principal topological space which does not have a $G$-point.

Next, we describe $G$-points in principal topological spaces. It is not hard to verify the following proposition.

Proposition 6.1. Let $X$ be a set equipped with a quasiordering $\Re$ and $a \in X$. The following statements are equivalent:

(1) $a$ is a $G$-point in $\left(X, T_{l}(\mathscr{R})\right)$;

(2) $a=b$ whenever $b \in X$ is such that $a \Re b$ and $b \mathscr{R} a$ (i.e., $\mathscr{R}_{l}(a) \cap \Re_{r}(a)=$ $\{a\})$.

EXAMPLE 6.2 (a principal topological space without any $G$-point). Take $X=$ $\mathbb{Z}$ equipped with the binary relation $\mathscr{R}$ defined by $m \mathscr{R} n \Leftrightarrow(m \leq n)$ or $(n$ is even and $n=m-1$ ).

If we equip $X$ with the left- $\mathscr{R}$-topology, then, in view of Proposition 6.1, $\operatorname{Gold}(X)$ is empty.

EXAMPLE 6.3 (a topological space $X$ which is Noetherian, infinite, and such that $\operatorname{Gold}(X)=X)$. Consider the set $X=\{0\} \cup\left\{1 / n \mid n \in \mathbb{N}^{*}\right\}$. Then $X$ equipped with the left topology associated to the natural ordering is an infinite Noetherian space such that $\operatorname{Gold}(X)=X$.

(i) Since $F=\left\{1 / n \mid n \in \mathbb{N}^{*}\right\}$ has no smallest element, $X$ is not partially well ordered.

(ii) We remark also that the $G$-topology is the discrete topology and since $X$ is infinite, the $G$-topology on $X$ is not quasicompact.

(iii) We notice that $(F, \geq)$ is not inductive.

(iv) $F$ is a $G$-closed irreducible subset of $X$ without generic point. Hence $F$ is not sober.

Note that the next examples are spaces of leaves classes. Nevertheless, we describe them from a topological point of view.

EXAMPLE 6.4 (a nonspectral topological space satisfying $(*)$ ). Let $X$ be the subset of $\mathbb{R}^{2}$ defined by $X=\{a\} \cup S$, where $a=(-2,0)$ and $S=\left\{(x, y) \in \mathbb{R}^{2} \mid\right.$ $\left.x^{2}+y^{2}=1\right\}$.

Consider on $X$ the topology $T$ such that $X$ is the only open subset of $X$ containing $a$ and such that for every $b \in S$, the family $\{B(b, \varepsilon) \cap S \mid \varepsilon>0\}$ is a base of neighborhoods of $b$ (where $B(b, \varepsilon)$ is the open ball of center $b$ and ray $\varepsilon$ ). This topology is compatible with the partial ordering $\leq$ defined on $X$ as follows: $b \leq b$ for every $b \in X$ and $a \leq b$ for every $b \in X$. The space $(X, T)$ satisfies property $(*)$ but is not spectral since $(X, T)$ has no base of quasicompact open subsets. 
EXAMPLE 6.5 (a topological space showing that the total ordering hypothesis in Proposition 3.16 is essential). (1) Let $R$ be an integral domain with finite dimension and $X=\operatorname{Spec}(R)$. Assume that $R$ is not a $G$-domain. Then the second statement of Proposition 3.16 holds while the first statement fails.

(2) Let $X=\{(0,1)\} \cup\{(0,0)\} \cup\left\{(1 / n, 0) \mid n \in \mathbb{Z}^{*}\right\}$ and set $a=(0,1), a_{\omega}=$ $(0,0)$, and $a_{n}=(1 / n, 0)$ if $n \neq 0$. We equip $X$ with the partial ordering $x \leq x$ and $x \leq a$ for each $x \in X$.

The family $\mathscr{B}=\{] \downarrow x]\}_{x \in X \sim a_{\omega}} \cup\left(\cup_{n \in Z^{*}}\left\{a_{\omega}\right\} \cup\left\{a_{p}|| p|\geq| n \mid\right\}\right)$ is a base for a topology $T$ compatible with the partial ordering $\leq$. We have $\operatorname{Gold}(X)=X$, but the topology $T$ is not the left topology.

\section{REFERENCES}

[1] E. Bouacida, O. Echi, and E. Salhi, Topologies associées à une relation binaire et relation binaire spectrale [Topologies associated with a binary relation and spectral binary relation], Boll. Un. Mat. Ital. B (7) 10 (1996), no. 2, 417-439 (French).

[2] _ Nonfinite heights, Commutative Ring Theory (Fès, 1995), Lecture Notes in Pure and Appl. Math., vol. 185, Marcel Dekker, New York, 1997, pp. 113130.

[3] _ Feuilletages et topologie spectrale [Foliations and spectral topology], J. Math. Soc. Japan 52 (2000), no. 2, 447-464 (French).

[4] A. Conte, Proprietà di separazione della topologia di Zariski di uno schema, Istit. Lombardo Accad. Sci. Lett. Rend. A 106 (1972), 79-111 (Italian).

[5] J. Dugundji, An extension of Tietze's theorem, Pacific J. Math. 1 (1951), 353-367.

[6] O. Echi, A topological characterization of the Goldman prime spectrum of a commutative ring, Comm. Algebra 28 (2000), no. 5, 2329-2337.

[7] M. Fontana and P. Maroscia, Sur les anneaux de Goldman, Boll. Un. Mat. Ital. B (5) 13 (1976), no. 3, 743-759 (French).

[8] O. Goldman, Hilbert rings and the Hilbert Nullstellensatz, Math. Z. 54 (1951), 136140.

[9] A. Grothendieck and J. Dieudonné, Eléments de Géométrie Algébrique, SpringerVerlag, Heidelberg, 1971.

[10] M. Hochster, Prime ideal structure in commutative rings, Trans. Amer. Math. Soc. 142 (1969), 43-60.

[11] _ The minimal prime spectrum of a commutative ring, Canad. J. Math. 23 (1971), 749-758.

[12] I. Kaplansky, Commutative Rings, revised ed., The University of Chicago Press, Illinois, 1974.

[13] W. Krull, Jacobsonsche Ringe, Hilbertscher Nullstellensatz, Dimensionstheorie, Math. Z. 54 (1951), 354-387 (German).

[14] G. Picavet, Autour des idéaux premiers de Goldman d'un anneau commutatif, Ann. Sci. Univ. Clermont Math. 57 (1975), no. 11, 73-90 (French).

[15] _ Sur les anneaux commutatifs dont tout idéal premier est de Goldman, C. R. Acad. Sci. Paris Sér. I Math. 280 (1975), no. 25, Ai, A1719-A1721 (French).

[16] R. Ramaswamy and T. M. Viswanathan, Overring properties of G-domains, Proc. Amer. Math. Soc. 58 (1976), 59-66.

[17] E. Salhi, Problème de structure dans les feuilletages de codimension un de classe $C^{0}$, thèse d'Etat, IRMA, Strasbourg, France, 1984. 
[18] A. K. Steiner, The lattice of topologies: structure and complementation, Trans. Amer. Math. Soc. 122 (1966), 379-398.

Ezzeddine Bouacida: Département de Mathématiques, Faculté des Sciences de Sfax, Université de Sfax, BP 802, 3018 Sfax, Tunisia

E-mail address: ezzedine.bouacida@fss.rnu.tn

Othman Echi: Department of Mathematics, Faculty of Sciences of Tunis, University Tunis-El Manar, “Campus Universitaire," 1092 Tunis, Tunisia

E-mail address: othman.echi@fst.rnu.tn

Gabriel Picavet: Laboratoire de Mathématiques Pures, Université Blaise Pascal, Complexe Scientifique des Cézeaux, 63177 Aubière Cedex, France

E-mail address: Gabrie1 . Picavet@math . univ-bpclermont. fr

Ezzeddine Salhi: Département de Mathématiques, Faculté des Sciences de Sfax, Université de Sfax, BP 802, 3018 Sfax, Tunisia

E-mail address: ezzeddine.salhi@fss.rnu.tn 


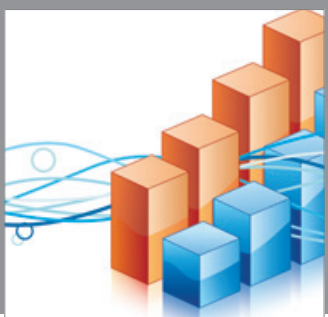

Advances in

Operations Research

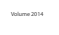

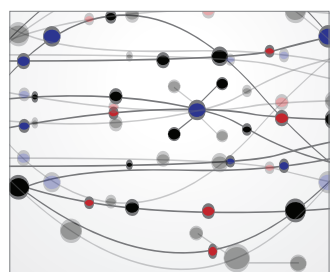

\section{The Scientific} World Journal
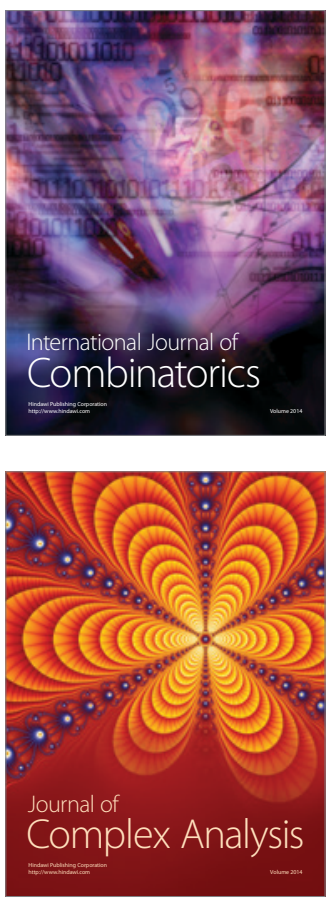

International Journal of

Mathematics and

Mathematical

Sciences
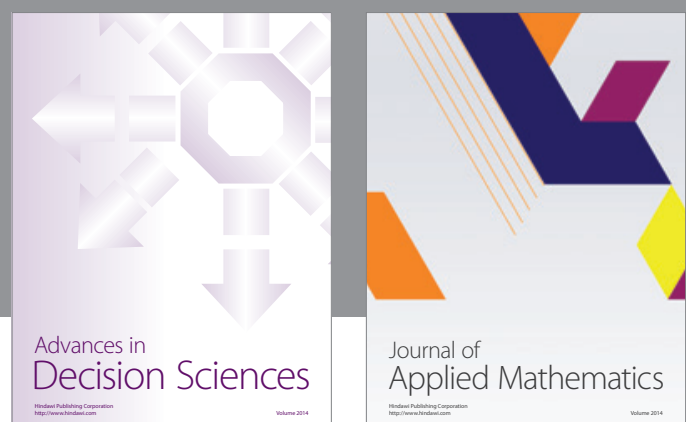

Journal of

Applied Mathematics
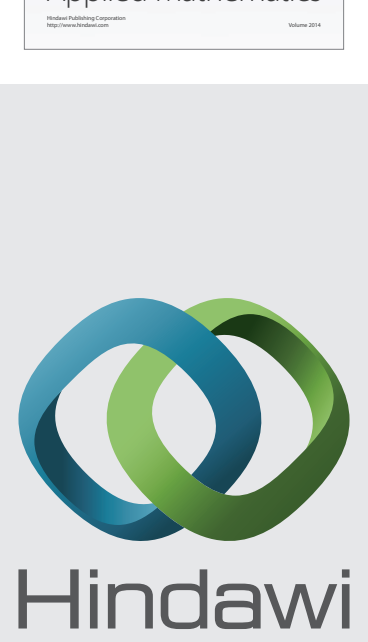

Submit your manuscripts at http://www.hindawi.com
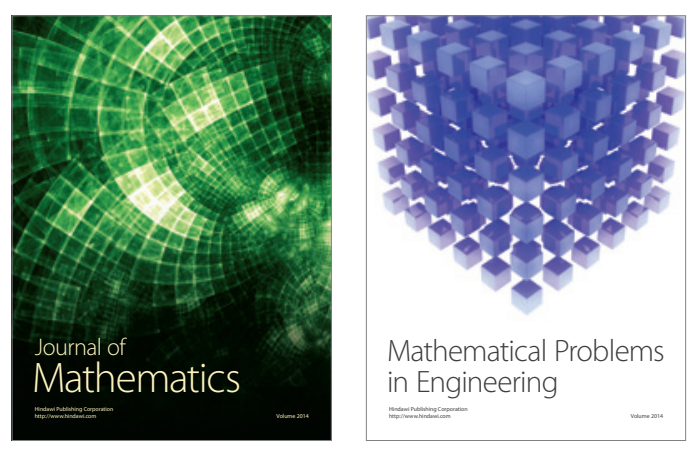

Mathematical Problems in Engineering
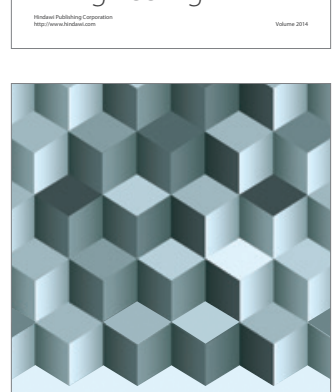

Journal of

Function Spaces
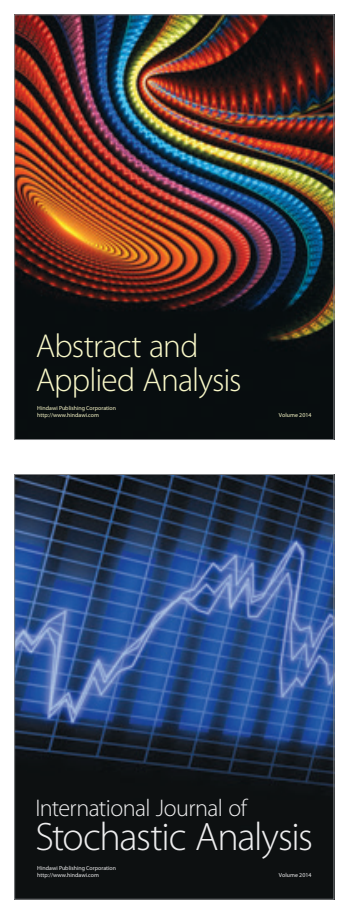

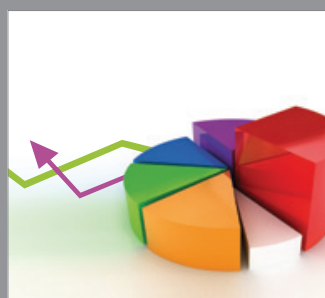

ournal of

Probability and Statistics

Promensencen
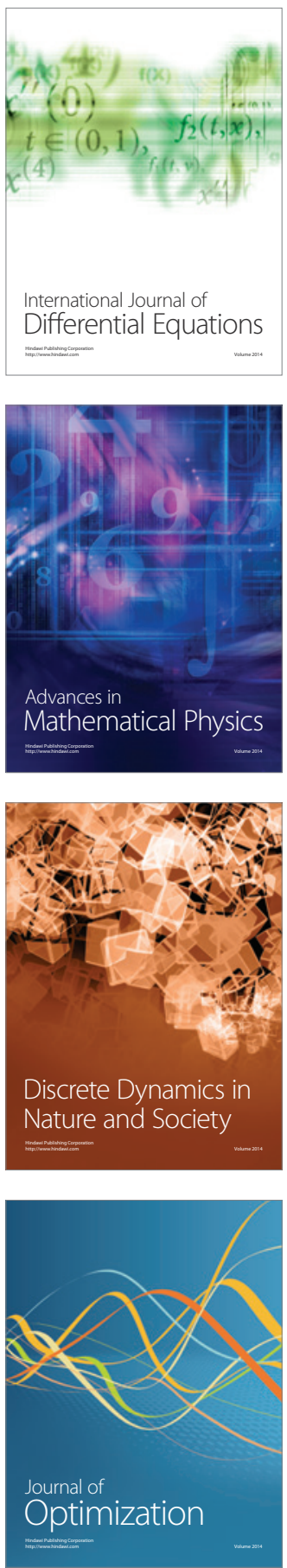